\title{
A model for pressurized hydrogen induced thin film blisters
}

R. A. J. M. van den Bos, V. Reshetniak, C. J. Lee, J. Benschop, and F. Bijkerk

Citation: Journal of Applied Physics 120, 235304 (2016); doi: 10.1063/1.4972221

View online: https://doi.org/10.1063/1.4972221

View Table of Contents: http://aip.scitation.org/toc/jap/120/23

Published by the American Institute of Physics

\section{Articles you may be interested in}

Band offsets and trap-related electron transitions at interfaces of (100)InAs with atomic-layer deposited $\mathrm{Al}_{2} \mathrm{O}_{3}$ Journal of Applied Physics 120, 235701 (2016); 10.1063/1.4971178

Interband and intraband relaxation dynamics in InSb based quantum wells

Journal of Applied Physics 120, 235702 (2016); 10.1063/1.4971347

Surface sensitivity of secondary electrons emitted from amorphous solids: Calculation of mean escape depth by a Monte Carlo method

Journal of Applied Physics 120, 235102 (2016); 10.1063/1.4972196

Strain-tunable ferroelectricity and its control of Rashba effect in $\mathrm{KTaO}_{3}$

Journal of Applied Physics 120, 234101 (2016); 10.1063/1.4972198

Reporting buckling strength and elastic properties of nanowires

Journal of Applied Physics 120, 235104 (2016); 10.1063/1.4972175

Spectral tailoring of nanoscale EUV and soft x-ray multilayer optics

Applied Physics Reviews 4, 011104 (2017); 10.1063/1.4978290

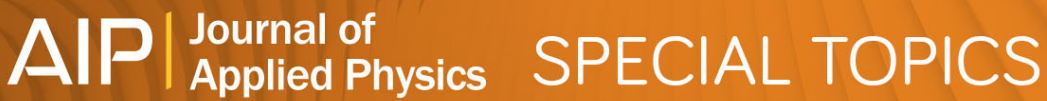

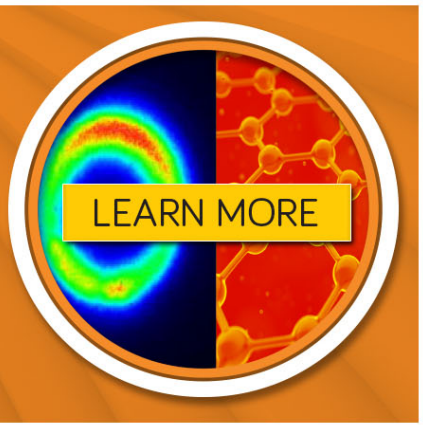




\title{
A model for pressurized hydrogen induced thin film blisters
}

\author{
R. A. J. M. van den Bos, ${ }^{1, a)}$ V. Reshetniak, ${ }^{2}$ C. J. Lee, ${ }^{1}$ J. Benschop,,${ }^{1,3}$ and F. Bijkerk ${ }^{1}$ \\ ${ }^{1}$ Industrial Focus Group XUV Optics, MESA+ Institute for Nanotechnology, University of Twente, Enschede, \\ The Netherlands \\ ${ }^{2}$ Institute for Spectroscopy Russian Academy of Sciences (ISAN), Troitsk, Moscow, Russian Federation \\ ${ }^{3}$ ASML Netherlands B.V., Veldhoven, The Netherlands
}

(Received 6 September 2016; accepted 1 December 2016; published online 16 December 2016)

\begin{abstract}
We introduce a model for hydrogen induced blister formation in nanometer thick thin films. The model assumes that molecular hydrogen gets trapped under a circular blister cap causing it to deflect elastically outward until a stable blister is formed. In the first part, the energy balance required for a stable blister is calculated. From this model, the adhesion energy of the blister cap, the internal pressure, and the critical H-dose for blister formation can be calculated. In the second part, the flux balance required for a blister to grow to a stable size is calculated. The model is applied to blisters formed in a Mo/Si multilayer after being exposed to hydrogen ions. From the model, the adhesion energy of the Mo/Si blister cap was calculated to be around $1.05 \mathrm{~J} / \mathrm{m}^{2}$ with internal pressures in the range of $175-280 \mathrm{MPa}$. Based on the model, a minimum ion dose for the onset of blister formation was calculated to be $d=4.2 \times 10^{18}$ ions $/ \mathrm{cm}^{2}$. From the flux balance equations, the diffusion constant for the Mo/Si blister cap was estimated to be $D_{H_{2}}=(10 \pm 1) \times 10^{-18} \mathrm{~cm}^{2} / \mathrm{s}$. Published by AIP Publishing. [http://dx.doi.org/10.1063/1.4972221]
\end{abstract}

\section{INTRODUCTION}

Nanometer thick multilayer structures can be designed and fabricated to form an artificial Bragg structure that can be used to reflect light of a specific wavelength. These mirrors can be found in synchrotrons, telescopes, and extreme ultraviolet optical systems. ${ }^{1,2}$ In many cases, the surfaces of such mirrors are exposed to fluxes of ionic and/or atomic hydrogen. This may be on purpose, for example, to remove contaminants from the mirror's surface to maintain optimal reflectivity., Exposure may also be due to the environmental conditions, as in the case of telescopes operating near planets and in the heliosphere. ${ }^{1}$ Although exposure to hydrogen can be beneficial for the multilayer optics, earlier investigations have shown that, under certain hydrogen exposure conditions, surface blisters may appear, which irreversibly damage the mirror surface. ${ }^{1,5}$

Blister formation is not exclusively related to multilayer mirrors but can also be found in a much broader research field, for example, in fusion reactor wall studies and the smart-cut process for silicon on insulator fabrication. ${ }^{6-11}$ Blisters have been observed in both heterogeneous nanometer thick layered structures, and also in bulk materials. In addition to hydrogen, helium ions have been found to induce blistering. ${ }^{12}$ Based on the experimentally observed blisters, several models have been developed to predict the critical dose for the onset of blister formation, adhesion energy, and radius of the blisters. ${ }^{13-16}$ In general, these models are based on the calculation of the potential energy of the blister cap as a function of pressure, volume, and elastic constants of the cap material. When the strain energy of deformation plus the surface energy is balanced by the mechanical work of the gas trapped inside the blister, a stable blister cap is formed. Besides models based on potential energy calculations,

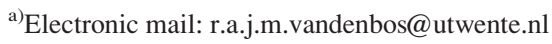

blister formation has been discussed in the framework of Föppl-von Karman theory, and finite element simulations. ${ }^{17-19}$ In these models, the coupling between internal pressure and the intrinsic stress in the layer is discussed in terms of buckling. In the works of Parry et al. ${ }^{17}$ and Coupeau et al., ${ }^{18,19}$ blisters were observed after additional compressive stress was applied to the layer, through increasing temperature, or externally applied mechanical force.

In this article, a blister formation model, based on pressure driven elastic deformation, is introduced. Special attention is paid to blisters formed in a Mo/Si multilayer by hydrogen ions, of which examples are shown in Figure 1. Here, we extend the previously described potential energy models in the following way. The compressive stress introduced during deposition is taken into account and in place of the ideal gas law an empirical equation of state (EOS), suitable for high pressure is used. Furthermore, we use the stable blister size to estimate the diffusion of molecular hydrogen through the blister cap. We show that the model agrees with experimental data. Finally, the model predictions for the influence of initial intrinsic stress, the adhesion energy, the

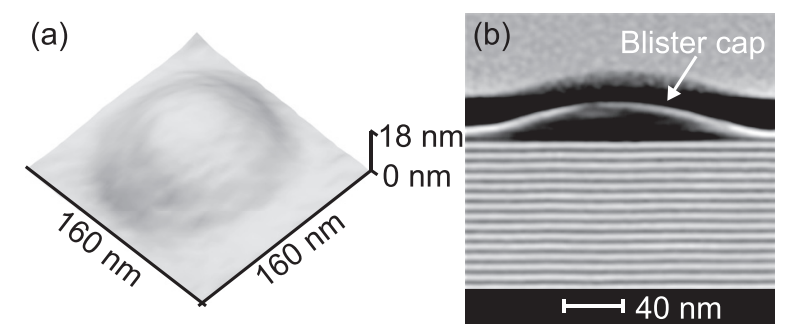

FIG. 1. Two examples of surface blisters formed on a Mo/Si multilayer after exposure to $200 \mathrm{eV}$ hydrogen ions. AFM image (a) and a cross sectional TEM image (b). The TEM image shows a delamination of the first $\mathrm{Mo} / \mathrm{Si}$ bilayer (Mo bright, Si dark). 
blister's internal pressure, and a minimum hydrogen dose for the onset of blisters are discussed.

\section{THEORY}

Blister formation is a multi-step mechanism that can qualitatively be described by the following steps: (i) Atomic and ionic hydrogen penetrates into the subsurface region of the thin film, either by direct ion implantation and/or diffusion; (ii) Because the solubility of hydrogen in the target material is limited, hydrogen segregates into micro cavities and defect sites, where it can recombine to molecular hydrogen and gets trapped; (iii) The pressure inside the cavity increases as more molecular hydrogen is accumulated up to the point where a blister is formed. (iv) The blister either stops growing or bursts depending on the transport of hydrogen through the material. ${ }^{20}$

To calculate the blister's energy balance, a blister shape must be assumed. Under the assumption that the blister cap can be described as an isotropic elastic thin film that deflects due to the pressure in the blister cavity, a stable blister size can be calculated depending on the number of trapped molecular hydrogen particles.

\section{A. Blister shape function}

A commonly used function for describing the blister shape is a bell shaped profile function as given by ${ }^{14,16,21}$

$$
z(r)= \begin{cases}z_{0}\left(1-\left(\frac{r}{r_{0}}\right)^{2}\right)^{2} & r \leq r_{0} \\ 0 & r>r_{0} .\end{cases}
$$

In this formula, $z(r)$ is the height of the blister cap at a distance $r$ from the blister top, which has a deflection $z_{0}$. The blister radius is given by $r_{0}$. This function is a solution of the classical plate equation from Poisson-Kirchhoff-Germain thin plate theory for small deflections ${ }^{22}$

$$
D \nabla^{2} \nabla^{2} z(r)=p,
$$

with $p$ as the pressure inside the blister and $D=$ $E t^{3}\left(12\left(1-\nu^{2}\right)\right)^{-1}$ as the plate constant. The plate constant is determined by the blister cap thickness $t$, Young's modulus $E$, and Poisson's ratio $\nu$. For a circular plate with fixed boundaries, i.e.,

$$
\begin{aligned}
& 2 D \frac{1}{r} \frac{d}{d r}\left[\frac{1}{r} \frac{d}{d r}\left(r \frac{d z(r)}{d r}\right)\right]=p, \\
& z\left(r_{0}\right)=0,\left.\quad \frac{d z(r)}{d r}\right|_{r=r_{0}}=0,
\end{aligned}
$$

the differential equation can be solved analytically to obtain Equation (1) with blister radius $r_{0}$, and a maximum deflection given by

$$
z_{0}=\frac{p r_{0}^{4}}{64 D}
$$

The above equation relates the blister shape to the internal pressure of the blister but only takes into account the bending moment of the blister. This means that for small deflections $z_{0} \ll t$ the blister height scales linearly with pressure. As will be shown in Sec. II B, a correction due to stretching should be taken into account for large deflections. The analytical solution presented in Equation (1) is fitted in Figure 2 (solid lines) to experimentally measured AFM profiles of $\mathrm{Mo} / \mathrm{Si}$ blister caps. To fit Equation (1), $z_{0}$ and $r_{0}$ are taken as free parameters. As can be seen in Figure 2, there is a good fit between the analytical shape function and the measured AFM profiles of the blister cap. The residual of the fit as given in the bottom graph is typically less than $8 \%$. The fit of Equation (1) overestimates the measured blister radius, which can be seen by the increase in the residual near the edge of the blister. This disagreement is likely due to local plastic deformation near the edge of the blister. ${ }^{23}$ In the model described in this article, plastic deformation is neglected.

\section{B. Energy balance of blister cap}

Several other calculations for the energy of the blister cap can be found in literature. For example, Selvadurai ${ }^{16}$ balanced the elastic strain energy including substrate deformation by the surface energy to determine the adhesion energy of thin films, while Freund ${ }^{13}$ and Hong and Cheong ${ }^{14}$ considered only the strain energy and surface energy to determine the minimal ion dose required for blisters to form.

For the blisters formed in a Mo/Si multilayer, the following equation is used to calculate the total potential energy of the blister cap

$$
E_{t o t}\left(z_{0}, r_{0}\right)=U_{b}+U_{s}+\Gamma+W_{e x p}+E_{i n t},
$$

in which the surface energy $\Gamma$, the elastic bending energy $U_{b}$, and the stretching energy $U_{s}$, are balanced by the expansion work done by the blister's internal pressure $W_{\text {exp }}$, and the release of intrinsic compressive stress energy $E_{\text {int }}$. The energy balance described by Equation (5) assumes that the deflection of the blister cap is small $\left(r_{0} \gg z_{0}\right)$ and the blister cap is thin

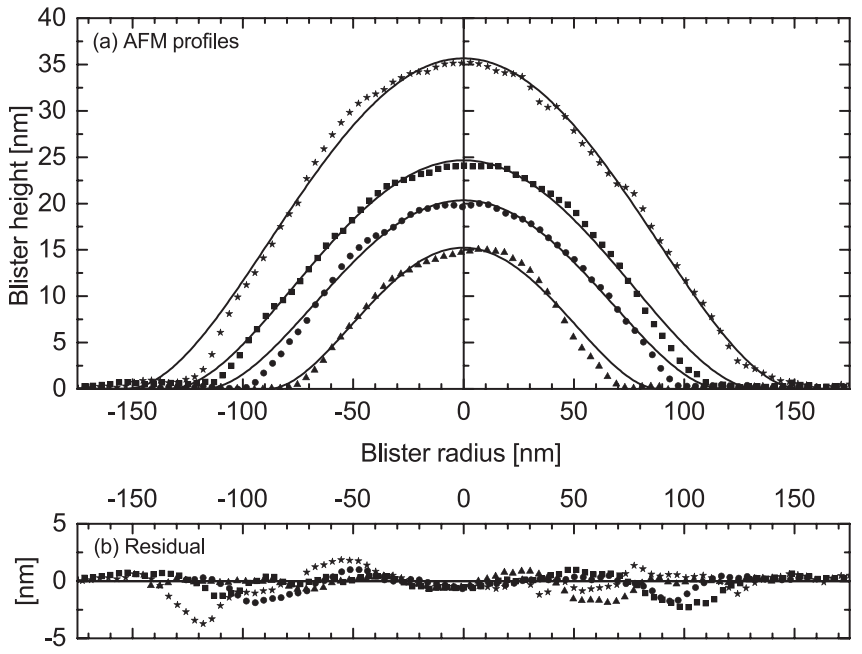

FIG. 2. Measured profiles of four blister caps (points) fitted with analytical expression of Equation (1) (solid lines). Bottom graph (b) shows residual of fitted function. 
$\left(r_{0} \gg t\right)$. As the observed deflection is comparable to the plate thickness $\left(z_{0} \approx 3 t\right)$, both stretching and bending are taken into account. To calculate $U_{b}$ and $U_{s}$, the blister cap is assumed to be isotropic and elastic with a shape given by Equation (1). The elastic constants are assumed not to change due to, e.g., hydrogen embrittlement. It is also assumed that the substrate is rigid, and therefore, the strain energy of the substrate is neglected. Dunders' coefficient for delamination at the Mo on $\mathrm{Si}$ interface $\left(\alpha_{d}=\left(\bar{E}_{f}-\bar{E}_{s}\right) /\left(\bar{E}_{f}+\bar{E}_{s}\right)\right.$ with $\bar{E}_{n}=E_{n}$ $\left./\left(1-\nu_{n}^{2}\right)\right)$ is estimated to be $\alpha_{d} \approx 0.45 .^{24-27}$ For $\alpha_{d} \approx 0.45$, Parry et al. $^{28}$ predicted that the blister's deflection is $15 \%$ more compared to a perfectly rigid substrate.

For small deflections $\left(z_{0}<r_{0}\right)$ of a thin film, the bending energy in cylindrical coordinates is defined $\mathrm{as}^{22}$

$$
\begin{aligned}
U_{b} & =\frac{1}{2} D \iint\left\{\left(\frac{\partial^{2} z}{\partial r^{2}}+\frac{1}{r} \frac{\partial z}{\partial r}\right)^{2}-2(1-\nu) \frac{\partial^{2} z}{\partial r^{2}}\left(\frac{1}{r} \frac{\partial^{2} z}{\partial r^{2}}\right)\right\} r d r d \theta \\
& =\frac{32}{3} \pi D\left(\frac{z_{0}}{r_{0}}\right)^{2} .
\end{aligned}
$$

If the deflection of the blister cap becomes comparable to the thickness, $z_{0}>t$, the stretching term, $U_{s}$, becomes significant. In this case, the in plane radial displacement $u(r)$ must be taken into account. Following the procedure of virtual displacement from Timoshenko and WoinowskyKrieger, ${ }^{22}$ and taking the radial displacement

$$
u(r)=r\left(r_{0}-r\right)\left(C_{1}+C_{2} r\right),
$$

the corresponding stretching energy is given by

$$
\begin{gathered}
U_{s}=\frac{\pi E t}{1-\nu^{2}} \int_{0}^{r_{0}}\left(\varepsilon_{r}^{2}+\varepsilon_{\theta}^{2}+2 \varepsilon_{r} \varepsilon_{\theta}\right) r d r \\
\varepsilon_{t}=\frac{\partial u}{\partial r}+\frac{1}{2}\left(\frac{\partial z}{\partial r}\right)^{2}, \quad \varepsilon_{\theta}=\frac{u}{r} .
\end{gathered}
$$

By minimizing the stretching energy, constants $C_{1}$ and $C_{2}$ can be calculated by taking the partial derivatives $\left(\partial U_{s}\right) /\left(\partial C_{1}\right)$ $=\left(\partial U_{s}\right) /\left(\partial C_{2}\right)=0$. This reduces Equation (8) to

$$
\begin{aligned}
& U_{s}=\frac{32}{3} \pi D\left(\frac{z_{0}}{r_{0}}\right)^{2}\left\{\frac{3}{32} C\left(\frac{z_{0}}{t}\right)^{2}\right\} \\
& C=\frac{-5582 \nu^{2}+8500 \nu+15010}{6615} .
\end{aligned}
$$

The surface energy released by the blister is given by the delaminated area as:

$$
\Gamma=2 \gamma \pi r_{0}^{2},
$$

with $\gamma$ the surface energy of the blister cap to substrate interface. In the model, a single value for the surface energy is assumed. In general, the adhesion energy changes as the blister grows in size, because changes in the mode mixity of the crack front appear. ${ }^{24}$ However, for high blister pressures $p_{n}=p_{E O S}\left\{\left(1-\nu^{2}\right) / E\right\}\left\{r_{0, e q} / t\right\}^{4}>1$ there are only minor changes in the mode mixity and the adhesion energy is, therefore, assumed to be constant.
The work done by isothermally expanding $n$ gas particles inside a blister cavity in terms of pressure and volume is

$$
W_{\text {exp }}=-\int_{V_{0}}^{V_{1}} p_{E O S}(V) d V+W_{0} .
$$

where $p_{E O S}(V)$ is the pressure as a function of volume, which is given by the equation of state (EOS). For large blisters, the EOS is simply the ideal gas law, but as the blister volume approaches zero, the gas significantly deviates from the ideal gas law due to particle interactions. Around the stationary point of the blister, the following EOS of state can be used, as found experimentally by Michels et al. ${ }^{29}$ for $\mathrm{H}_{2}$ pressures in the range of 2-300 MPa (Ref. 29)

$$
p_{E O S}(V)=A \frac{n}{V}\left\{1+B \frac{n}{V}+C \frac{n^{2}}{V^{2}}+D \frac{n^{3}}{V^{3}}+E \frac{n^{4}}{V^{4}}+F \frac{n^{5}}{V^{5}}\right\},
$$

with $n$ the number of particles in moles, $V$ the volume in cubic meter, and coefficients $A$ through $F$ as given in Table I. With the above EOS, the molar density approaches that of solid hydrogen for pressures around $300 \mathrm{MPa}$. For the expansion work, this leads to the equations

$$
\begin{gathered}
W_{\exp }=W\left(V_{1}\right)-W\left(V_{0}\right)+W_{0}, \\
W(V)=-A n\left\{\ln (V)-B \frac{n}{V}-\frac{1}{2} C \frac{n^{2}}{V^{2}}+\ldots\right\}, \\
V=\frac{1}{3} \pi r_{0}^{2} z_{0},
\end{gathered}
$$

where $W_{0}$ is the expansion work done for pressures above $300 \mathrm{MPa}$ and $W\left(V_{1}\right)-W\left(V_{0}\right)$ is the expansion work done for pressures within the validity range of the EOS. We assume that $W_{0}$ is constant for all blisters formed. The blister volume is calculated by taking the volume integral of Equation (1).

Depending on the deposition process of the multilayer, the average stress of the $\mathrm{Mo} / \mathrm{Si}$ bilayer can vary from hundreds of MPa pressure compressive to tensile. ${ }^{30}$ For an initially compressively stressed blister cap the energy released by the delaminated layer is given by

$$
E_{i n t}=-\frac{1-\nu}{E} \sigma_{i n t}^{2} t \pi r_{0}^{2}
$$

where $\sigma_{\text {int }}$ is the average compressive stress in the thin layer. If the film has a tensile stress the sign of the energy is changed and additional energy needs to be added to deflect the surface outward. As clamped blister cap conditions are

TABLE I. Coefficients for Equation of State (EOS) given in Equation (12) for a temperature $\mathrm{T}=298 \mathrm{~K}$.

\begin{tabular}{lccc}
\hline & Value & & Value \\
\hline $\mathrm{A}(=\mathrm{RT})$ & 2479.62 & $\mathrm{D}$ & $3.3804 \times 10^{-15}$ \\
$\mathrm{~B}$ & $1.4384 \times 10^{-5}$ & $\mathrm{E}$ & $9.2492 \times 10^{-20}$ \\
$\mathrm{C}$ & $3.5637 \times 10^{-10}$ & $\mathrm{~F}$ & $-4.7594 \times 10^{-25}$ \\
\hline \hline
\end{tabular}


TABLE II. Material constants and dimensions used to model the blister cap in a $\mathrm{Mo} / \mathrm{Si}$ multilayer.

\begin{tabular}{lc}
\hline \hline Parameter & Value \\
\hline$E$ & $215 \mathrm{GPa}^{\mathrm{a}}$ \\
$\nu$ & $0.18^{\mathrm{a}}$ \\
$\gamma$ & $1.05 \mathrm{~J} / \mathrm{m}^{2}$ \\
$\sigma_{\text {int }}$ & $500 \mathrm{MPa}$ \\
$t$ & $7 \mathrm{~nm}$ \\
\hline \hline
\end{tabular}

${ }^{\mathrm{a}}$ Calculated values taken from Loopstra et al. ${ }^{31}$

assumed (no radial displacement and rotation of the blister edge), Equation (14) only considers the strain energy stored within the delaminated area $\left(r<r_{0}\right)$. One can show that for a deposited multilayer with material parameters as shown in Table II, the intrinsic stress has only a minor effect on the energy balance. (Compared to $U_{b}$ and $U_{s}$, the intrinsic stress is about two orders of magnitude lower, $\approx 10^{-14} \mathrm{~J}$ compared to $\approx 10^{-16} \mathrm{~J}$ ).

Adding all energy terms as given in Equation (5), the total energy of the blister cap as a function of blister radius and height can be found for a fixed number of $n$ hydrogen particles inside the blister cavity. For a Mo/Si multilayer, the contour lines of the energy surface for $15 \times 10^{6}$ trapped particles are shown in Figure 3. In this calculation, material constants and dimensions are used as shown in Table II.

In the white area, the pressure inside the blister exceeds $300 \mathrm{MPa}$. At that pressure, the hydrogen density approaches that of solid hydrogen and the expansion work can no longer be calculated from the EOS. For the limiting case, it can be seen that as the volume goes to zero, the expansion work tends to infinity. On the other hand, the surface energy (blister radius) and stretching energy (blister height) will increase continuously for an increasing blister size. Thus, for a fixed number of trapped molecular hydrogen inside the blister, a stable minimum in the blister cap energy can be found, as indicated by the red arrow in Figure 3.

\section{Stable blister shape}

To find the stable point as shown in Figure 3, the partial derivatives of $E_{\text {tot }}$ with respect to $r_{0}$ and $z_{0}$ are taken. This leads to the following equations for the stable point:

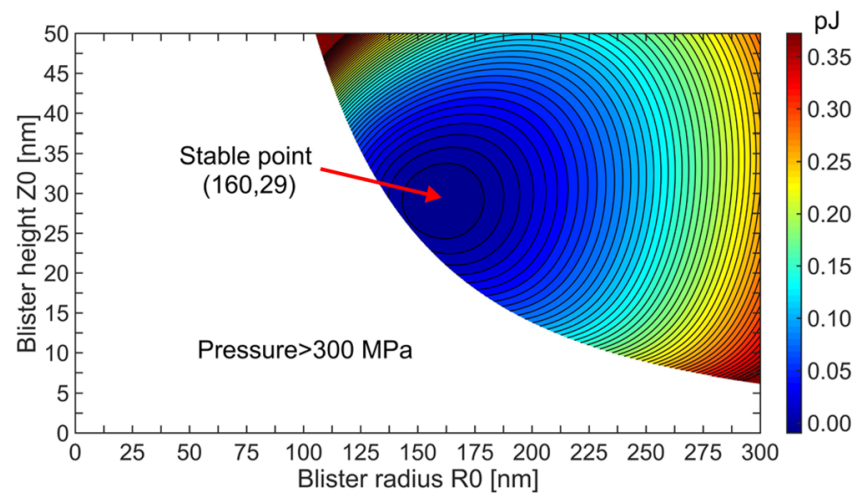

FIG. 3. Contour plot of $E_{t o t}\left(z_{0}, r_{0}\right)$ for 15 million trapped hydrogen particles. At $(160,29)$ a stable minimum is found for the total energy of the blister cap.

$$
\begin{gathered}
z_{0, e q}=\frac{p_{E O S}\left(n, r_{0, e q}, z_{0, e q}\right) r_{0, e q}^{4}}{64 D} \frac{1}{1+\frac{3}{16} C\left(\frac{z_{0, e q}}{t}\right)^{2}}, \\
r_{0, e q}=\sqrt[4]{\frac{16 D z_{0, e q}^{2}}{\gamma-\frac{1-\nu}{2 E} \sigma_{i n t}^{2} t}\left\{1+\frac{5}{32} C\left(\frac{z_{0, e q}}{t}\right)^{2}\right\} .}
\end{gathered}
$$

The first equation relates the blister's internal pressure to its dimensions $r_{0}$ and $z_{0}$. It is comparable with Equation (4) but an additional term is included that takes the stretching of the blister cap into account. With increasing number of particles, the stable blister size increases. The second equation gives the minimum in the blister cap energy surface. If $z_{0, e q} \gg t$, there is a linear dependence between the blister radius and blister height. In Figure 4, the stable blister dimensions for four different surface energies are calculated taken the values as given in Table II. It can be seen that for increasing surface energies the ratio between blister height and radius increases. To verify the model, data are taken from an atomic force microscope measurement on a blistered $\mathrm{Mo} / \mathrm{Si}$ multilayer surface being exposed to hydrogen. From the graph, it is seen that the surface energy of the delaminated surface is around $1.05 \mathrm{~J} / \mathrm{m}^{2}$ which equals the surface energy of a-Si as can be found in literature: $1.05 \pm 0.14 \mathrm{~J} / \mathrm{m}^{2}{ }^{32}$ For comparison, the surface energy of (001) Mo and (001) $\mathrm{MoSi}_{2}$ is around $3.97 \mathrm{~J} / \mathrm{m}^{2}$ and $3.86 \mathrm{~J} / \mathrm{m}^{2}$, respectively. ${ }^{33}$ So, based on the model, it is expected that the delamination has taken place within the a-Si layer.

For a stable blister, both Equations (15a) and (15b) have to be satisfied. When both equations are combined and dimensionless constants are introduced, the following relation between internal pressure $p_{E O S}$, intrinsic stress $\sigma_{\text {int }}$, and surface energy $\gamma$ is obtained:

$$
\begin{gathered}
p_{n}=z_{n} \zeta \sqrt{24 \gamma_{n}-\frac{12}{1+\nu} \sigma_{n}^{2}}, \\
\zeta=\frac{\frac{16}{3}+C z_{n}^{2}}{\sqrt{32 z_{n}^{2}+5 C z_{n}^{2}}},
\end{gathered}
$$

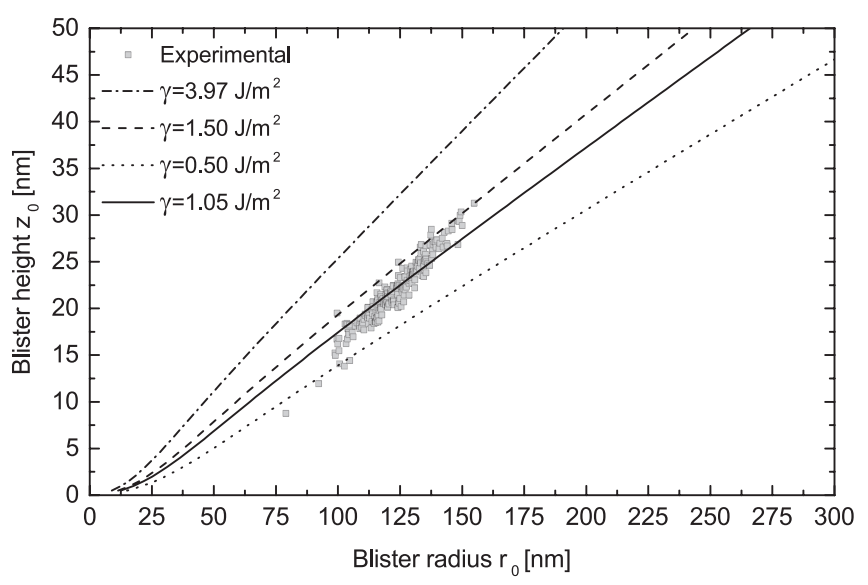

FIG. 4. Equilibrium of blister radius and height as a function for four different surface energies. The experimental data is an AFM measurement of blisters formed on a Mo/Si multilayer after hydrogen exposure. 
with $z_{n}=z_{0, e q} t^{-1}, \quad r_{n}=r_{0, e q} t^{-1}, \quad \bar{E}=E\left(1-\nu^{2}\right)^{-1}, p_{n}$ $=r_{n}^{4} p_{E O S} \bar{E}^{-1}, \quad \gamma_{n}=r_{n}^{4} \gamma(\bar{E} t)^{-1}$, and $\sigma_{n}=r_{n}^{2} \sigma_{\text {int }} \bar{E}^{-1}$. From Equation (16), it can be seen that the internal pressure decreases with increasing layer stress, as expected from Parry et al. ${ }^{17}$

\section{Hydrogen density and pressure inside a Mo/Si multilayer blister}

From a measured blister shape, the hydrogen density and pressure inside the blister can be calculated with Equations (15) and (12). In Figure 5, the blister pressure and density are given as a function of the stable blister radius. For blister radii smaller than $\approx 90 \mathrm{~nm}$, the hydrogen density necessary for stable blisters to form approach values of solid hydrogen (dotted line). The dashed dotted line indicates the measured blister radius range and the corresponding range in blister density $\left(20.1-25.3 \mathrm{H}_{2} / \mathrm{nm}^{3}\right)$ and pressure (175-280 MPa).

When the stable blister shape and density are known, an estimate can be made on the minimum dose required to form the blister. For an observed blister with a radius of $98 \mathrm{~nm}$ and corresponding height of $17 \mathrm{~nm}$, the local hydrogen density has to be $25.3 \mathrm{H}_{2} / \mathrm{nm}^{3}$. This means that $N_{\text {min }}=4.3$ million particles have to get trapped in the blister cavity. The number of hydrogen particles reaching the blister volume $N_{\text {min }}$ is given by

$$
N_{\text {min }}=\frac{1}{2} f d \pi r_{0}^{2}
$$

In this equation, $d$ is the incident hydrogen ion dose per unit area and $f$ is the fraction of the incoming ions that can penetrate through the blister cap. The factor of one half takes into account the recombination of hydrogen ions to stable molecular hydrogen. In this equation, the diffusion of hydrogen after implantation is neglected. To estimate $f$, an SRIM calculation was performed for a Mo/Si multilayer irradiated by $100 \mathrm{eV}$ hydrogen ions (see Figure 6). ${ }^{34,35}$ The fraction of the total flux that can penetrate through the first bilayer is $f \approx 6.8 \times 10^{-3}$. Filling the number in Equation (17) gives a minimum required

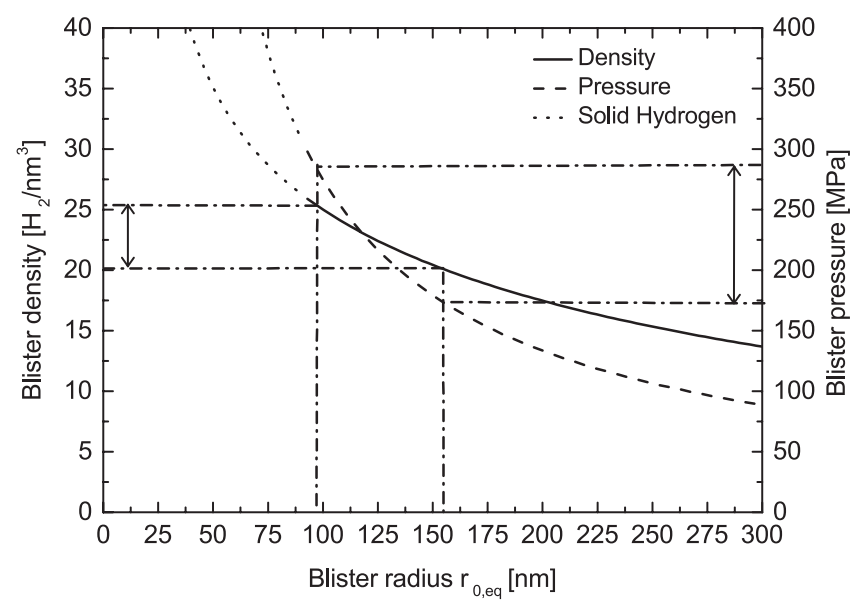

FIG. 5. Blister pressure and density as a function of the stable blister radius. The dashed dotted line indicates the experimental measured blister radii and their corresponding ranges for the pressure and density. ion dose of $d=4.2 \times 10^{18}$ ions $/ \mathrm{cm}^{2}$. This is indeed below the actual measurement dose of $1.25 \times 10^{19}$ ions $/ \mathrm{cm}^{2}$.

Equation (17) accounts for ions that directly penetrate through the blister cap, but neglects any hydrogen diffusion. This is justified because the estimated timescale of hydrogen diffusion is long (hours order of magnitude, see Section IIE)), compared to the timescale of blister formation.

\section{E. Blister stabilization}

In the analysis above, only the static case of the blister is considered where the number of trapped hydrogen particles inside the blister is fixed. But in general, depending on the in- and outflux of hydrogen $\left(H_{i n}, H_{\text {out }}\right)$, three cases can be distinguished: (i) $H_{\text {in }}>H_{\text {out }}$ : the number of trapped hydrogen particles is increasing and the blister grows; (ii) $H_{\text {in }}=H_{\text {out }}$ : the number of trapped hydrogen particles is fixed and the blister is stable at its energetically most favorable shape; (iii) $H_{\text {in }}<H_{\text {out }}$ : the number of trapped hydrogen particles is decreasing and the blister size decreases assuming the deformation is completely elastic. By knowing the inand outflux as a function of time, the dynamic behavior of the blister can be described.

The influx of hydrogen per unit of time $\mathrm{H}_{\text {in }} \mathrm{H}_{2} / \mathrm{s}$ is given by

$$
H_{\text {in }}=\frac{1}{2} \phi f \pi r_{0}^{2}
$$

with $\phi$ ions $/ \mathrm{cm}^{2}$ s the hydrogen ion flux at the surface and $f$ is the fraction of ions that can penetrate through the blister cap. The out diffusion $H_{\text {out }} \mathrm{H}_{2} / \mathrm{s}$ of hydrogen can be estimated using Fick's law

$$
H_{\text {out }}=D_{H_{2}} \frac{n / V}{t} S_{\text {blister }}
$$

The diffusion constant $D_{H_{2}} \mathrm{~cm}^{2} / \mathrm{s}$ depends on the blister cap material and $n$ is the number of hydrogen particles that are trapped inside the blister with a volume $V$. $S_{\text {blister }}$ is the blister cap surface area

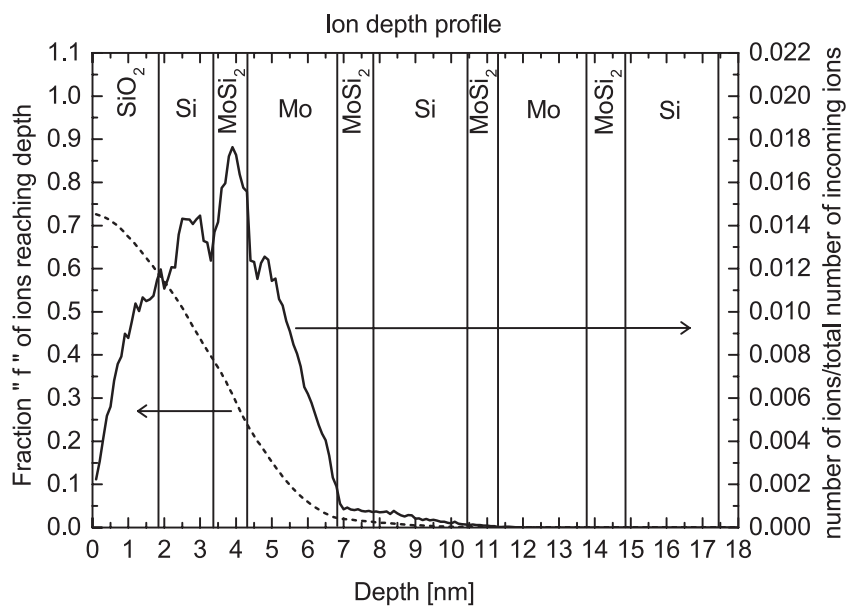

FIG. 6. SRIM calculation of hydrogen ion penetration depth in a Mo/Si multilayer with native $\mathrm{SiO}_{2}$ on top. Fraction $f$ of total flux that reaches a certain depth (dashed line) and number of ions at certain depth (solid line) are given. 


$$
\begin{gathered}
S_{\text {blister }}=\iint z(r) d S \approx \pi r_{0}^{2} F . \\
F=1-0.0036\left(\frac{z_{0}}{r_{0}}\right)+0.715\left(\frac{z_{0}}{r_{0}}\right)^{2}-0.205\left(\frac{z_{0}}{r_{0}}\right)^{3} .
\end{gathered}
$$

From the final shape of the blister, the diffusion constant $D_{H_{2}}$ can be calculated by combining Equations (18) and (19)

$$
D_{H_{2}}=\frac{\phi f t V}{2 n} \frac{1}{F} \text {. }
$$

Taking the blister shape of the measured data with a number density between 20.1 and $25.3 \mathrm{H}_{2} / \mathrm{nm}^{3}$ exposed at a constant flux of $\phi=1 \times 10^{14}$ ions $/ \mathrm{cm}^{2} \mathrm{~s}$, the diffusion constant is around $D_{H_{2}}=(10 \pm 1) \times 10^{-18} \mathrm{~cm}^{2} / \mathrm{s}$. This is about an order of magnitude higher than the literature value for hydrogen diffusion in $\mathrm{c}-\mathrm{Si}{ }^{25} D_{\mathrm{H}_{2}}=2.36 \times 10^{-18} \mathrm{~cm}^{2} / \mathrm{s}$.

Before the blister is stabilized, the blister is growing which means that $H_{\text {in }}>H_{\text {out }}$. Because the influx of hydrogen particles per unit area is constant during the experiment, the outflux of hydrogen per unit area has to increase during the blister growth until the influx is balanced and the blister growth will stop. This means that regarding Equation (19), the hydrogen density n/V and/or the diffusion constant $D_{H_{2}}$ has to increase in time. At the start of the hydrogen exposure, the concentration inside the multilayer is zero and it is slowly increasing with time. While the influx is set instantaneously by the ion flux and energy, the outflux is increasing towards the influx value with a certain delay depending on the diffusion constant $D_{H_{2}}$ and thickness of the blister cap. The timescale for out diffusion can be estimated with

$$
\tau=\frac{t^{2}}{D_{H_{2}}} \approx 10^{4} s
$$

This is the typical time it takes for a hydrogen particle to diffuse through the blister cap. During this time, about $\tau \times H_{\text {in }}=4$ million $\mathrm{H}_{2}$ particles have penetrated through the blister cap. This is about the number of particles needed to form a stable blister of $100 \mathrm{~nm}$ in radius. For a large diffusion constant the delay between in and outflux becomes smaller and no blisters will form at all as not sufficient hydrogen can get trapped. On the other hand, a lower diffusion coefficient will increase the blister size as more particles get trapped before a balance between in and outflux is established.

Although it is possible to form a stable blister where fluxes are balanced, this point does not have good stability. As the blister radius increases beyond the stable point, the influx of hydrogen per unit area becomes larger than the outflux, and the blister can continue to grow until it bursts. This contradicts the stable blister shape observed on a Mo/Si multilayer. A possible explanation for this can be found in a time dependent diffusion constant. Studies on the hydrogenation of amorphous silicon have shown that the permeability of a-Si can change significantly during the hydrogenation. ${ }^{36}$ These studies suggest that before the Si layer is fully saturated, only atomic hydrogen can diffuse through the silicon layer and the rate of diffusion is rather low. However, once a-Si:H is fully hydrogenated, $\mathrm{H}_{2}$ can freely and rapidly diffuse through the layer. This means that during the blister formation process this enhanced outdiffusion of hydrogen may prevent further blister growth. The magnitude of the change in the diffusion constant required to prevent further blister growth depends on the (time-dependent) size of the blister. For a blister near its initiation point, a doubling in the diffusion constant may be enough to stop the blister growth process. In addition, Coupeau et al..$^{21}$ also suggested that changes in the diffusion process occur during blister formation. Coupeau et al. showed that the evolution of blisters on a pure silicon surface has small discontinuities over time, which the authors address to changes in the diffusion process.

\section{CONCLUSION}

The results presented here show that an elastic energy balance of a blister cap can be applied to hydrogen induced blister formation in $\mathrm{Mo} / \mathrm{Si}$ multilayer mirrors. The model shows that the blister formation is mainly caused by the accumulation of hydrogen under the blister cap and that a stable blister can be formed with a fixed number of particles inside. From the measured blister radius and height, the surface energy, pressure, and minimum hydrogen dose for blister formation could be calculated from the model. In the second part, the diffusion and penetration through the blister cap was considered. Given a fixed influx of hydrogen and assuming a linear outdiffusion of hydrogen, a diffusion constant for hydrogen through the blister cap could be calculated. Furthermore, the lack of blister expansion after the stable blister has been formed is likely caused by a time dependent permeability of the blister cap.

\section{ACKNOWLEDGMENTS}

The authors would like to thank Dimitry Lopaev and Slava Medvedev for their contributions to the scientific discussions about this model. This work was part of the research programme $\mathrm{CP} 3 \mathrm{E}$ (Controlling photon and plasma induced processes at EUV optical surfaces) of FOM (Stichting voor Fundamenteel Onderzoek der Materie) with the financial support of NWO (Nederlandse organisatie voor Wetenschappelijk Onderzoek). The CP3E programme is cofinanced by Carl Zeiss SMT and ASML, and AgentschapNL through the EXEPT programme.

${ }^{1}$ M. G. Pelizzo, A. J. Corso, P. Zuppella, D. L. Windt, G. Mattei, and P. Nicolosi, Opt. Express 19, 14838 (2011).

${ }^{2}$ C. Teyssier, E. Quesnel, V. Muffato, and P. Schiavone, Microelectron. Eng. 61-62, 241 (2002).

${ }^{3}$ K. Motai, H. Oizumi, S. Miyagaki, I. Nishiyama, A. Izumi, T. Ueno, and A. Namiki, Thin Solid Films 516, 839 (2008).

${ }^{4}$ M. Pachecka, J. M. Sturm, R. W. E. van de Kruijs, C. J. Lee, and F. Bijkerk, AIP Adv. 6, 075222 (2016).

${ }^{5}$ A. S. Kuznetsov, M. A. Gleeson, and F. Bijkerk, J. Appl. Phys. 114, 113507 (2013).

${ }^{6}$ J. H. Evans, J. Nucl. Mater. 61, 1 (1976).

${ }^{7}$ X. Q. Feng and Y. Huang, Int. J. Solids Struct. 41, 4299 (2004).

${ }^{8}$ T. Höchbauer, A. Misra, K. Hattar, and R. G. Hoagland, J. Appl. Phys. 98, 123516 (2005).

${ }^{9}$ S. Sharafat, A. Takahashi, K. Nagasawa, and N. Ghoniem, J. Nucl. Mater. 389, 203 (2009). 
${ }^{10}$ D. G. Xie, Z. J. Wang, J. Sun, J. Li, E. Ma, and Z. W. Shan, Nat. Mater. 14, 899 (2015).

${ }^{11}$ R. Singh, S. H. Christiansen, O. Moutanabbir, and U. Gösele, J. Electron. Mater. 39, 2177 (2010).

${ }^{12}$ P. B. Johnson, R. W. Thomson, and K. Reader, J. Nucl. Mater. 273, 117 (1999).

${ }^{13}$ L. B. Freund, Appl. Phys. Lett. 70, 3519 (1997).

${ }^{14}$ J. W. Hong and S. Cheong, J. Appl. Phys. 100, 094322 (2006).

${ }^{15}$ K. Mitani and U. M. Gösele, Appl. Phys. A: Solids Surfaces 54, 543 (1992).

${ }^{16}$ A. P. S. Selvadurai, Acta Mater. 55, 4679 (2007).

${ }^{17}$ G. Parry, C. Coupeau, E. Dion, M. L. David, J. Colin, and J. Grilhé, J. Appl. Phys. 110, 114903 (2011).

${ }^{18}$ C. Coupeau, J. Grilhé, E. Dion, L. D. de Morais, and J. Colin, Thin Solid Films 518, 5233 (2010)

${ }^{19}$ C. Coupeau, E. Dion, M. L. David, J. Colin, and J. Grilhé, Europhys. Lett. 92, 16001 (2010).

${ }^{20}$ B. Terreault, Phys. Status Solidi A 204, 2129 (2007).

${ }^{21}$ C. Coupeau, G. Parry, J. Colin, M. L. David, J. Labanowski, and J. Grilhé, Appl. Phys. Lett. 103, 031908 (2013).

${ }^{22}$ S. Timoshenko and S. Woinowsky-Krieger, Theory of Plates and Shells, 2nd ed. (McGraw-Hill, 1959).

${ }^{23}$ J. Colin, C. Coupeau, and J. Grilhé, Phys. Rev. Lett. 99, 046101 (2007).
${ }^{24}$ J. W. Hutchinson and Z. Suo, Adv. Appl. Mech. 29, 63-191 (1991).

${ }^{25}$ B. L. Sharma, CRC handbook of Chemistry and Physics (CRC Press, 2005), Chap. 12, internet version 2005.

${ }^{26}$ R. Kuschnereit, H. Fath, A. A. Kolomenskii, and M. Szabadi, Appl. Phys. A Mater. Sci. Process. 61, 269 (1995).

${ }^{27}$ M. A. Hopcroft, W. D. Nix, and T. W. Kenny, J. Microelectromech. Systems 19, 229 (2010).

${ }^{28}$ G. Parry, J. Colin, C. Coupeau, F. Foucher, A. Cimetière, and J. Grilhé, Acta Mater. 53, 441 (2005).

${ }^{29}$ A. Michels, W. de Graaff, T. Wassenaar, J. M. H. Levelt, and P. Louwerse, Physica 25, 25 (1959).

${ }^{30}$ D. L. Windt, W. L. Brown, C. A. Volkert, and W. K. Waskiewicz, J. Appl. Phys. 78, 2423 (1995).

${ }^{31}$ O. B. Loopstra, E. R. van Snek, T. H. de Keijser, and E. J. Mittemeijer, Phys. Rev. B 44, 13519 (1991).

${ }^{32}$ S. Hara, S. Izumi, T. Kumagai, and S. Sakai, Surf. Sci. 585, 17 (2005).

${ }^{33}$ T. Hong, J. R. Smith, D. J. Srolovitz, J. G. Gay, and R. Richter, Phys. Rev. B 45, 8775 (1992).

${ }^{34}$ J. Ziegler, J. Biersack, and U. Littmark, The Stopping and Range of Ions in Matter (Pergamon, New York, 1985).

${ }^{35}$ See http://www.srim.org/ for SRIM (last accessed July 1, 2016).

${ }^{36}$ W. Beyer, Phys. Status Solidi A 213, 1661 (2016). 